\section{Commentary: Children are not small adults: New parameters for pediatric Marfan syndrome}

\author{
Alvise Guariento, MD, ${ }^{\mathrm{a}}$ David Blitzer, MD, ${ }^{\mathrm{b}}$ and \\ Vladimiro Vida, $\mathrm{MD}, \mathrm{PhD}^{\mathrm{a}}$
}

The relatively small number of patients with congenital heart disease compared with those with acquired disease sometimes poses great challenges in finding the right parameters to guide medical and surgical management of this population. This is especially true when cardiologists and cardiac surgeons deal with patients with syndromic disease. Therefore, the simplest solution is to derive certain values in adults with syndromic disease and infer operative indications in children with the same syndrome. However, this could not be more wrong because children are not just small adults (Figure 1). ${ }^{1}$

Ahmed Bhimani and colleagues ${ }^{2}$ show how this concept applies perfectly to patients with Marfan syndrome. Indeed, the authors demonstrate how a relatively new parameter (aortic root cross-sectional area/height) tracked rapid somatic growth of these patients better than standard measures (eg, aortic root $z$ score). Beyond that, they brilliantly propose a threshold of 5 to $7 \mathrm{~cm}^{2} / \mathrm{m}$ as a potential limit for surgical consideration. It is probably this aspect on which the most important finding of their work is based: The surgical thresholds for pediatric patients with Marfan syndrome appear to be lower than those derived from a corresponding adult population.

Unfortunately, the findings of this article need to be confirmed in a larger population. In particular, surgical indications depend on the broadest possible verification, which should include several institutions with alternative protocols and approaches. Furthermore, studies assessing

\footnotetext{
From the ${ }^{\mathrm{a}}$ Pediatric and Congenital Cardiac Surgery Unit, Department of Cardiac, Thoracic, Vascular Sciences, and Public Health, University of Padua, Padua, Italy; and ${ }^{\mathrm{b}}$ Department of Surgery, Columbia University Medical Center, New York, NY. Disclosures: The authors reported no conflicts of interest.

The Journal policy requires editors and reviewers to disclose conflicts of interest and to decline handling or reviewing manuscripts for which they may have a conflict of interest. The editors and reviewers of this article have no conflicts of interest.

Received for publication Dec 21, 2021; revisions received Dec 21, 2021; accepted for publication Dec 22, 2021; available ahead of print Dec 27, 2021.

Address for reprints: Alvise Guariento, MD, Pediatric and Congenital Cardiac Surgery Unit, Department of Cardiac, Thoracic, Vascular Sciences, and Public Health, University of Padua, Via Giustiniani 2, 35100 Padua, Italy (E-mail: alvise. guariento@hotmail.com).

J Thorac Cardiovasc Surg 2022;164:741

$0022-5223 / \$ 36.00$

Copyright (c) 2022 by The American Association for Thoracic Surgery

https://doi.org/10.1016/j.jtcvs.2021.12.042
}

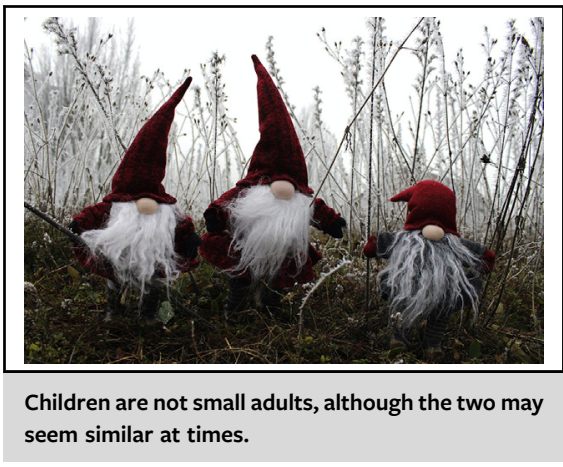

CENTRAL MESSAGE

New parameters are needed for the pediatric population with Marfan syndrome. These should not be derived from the adult population.

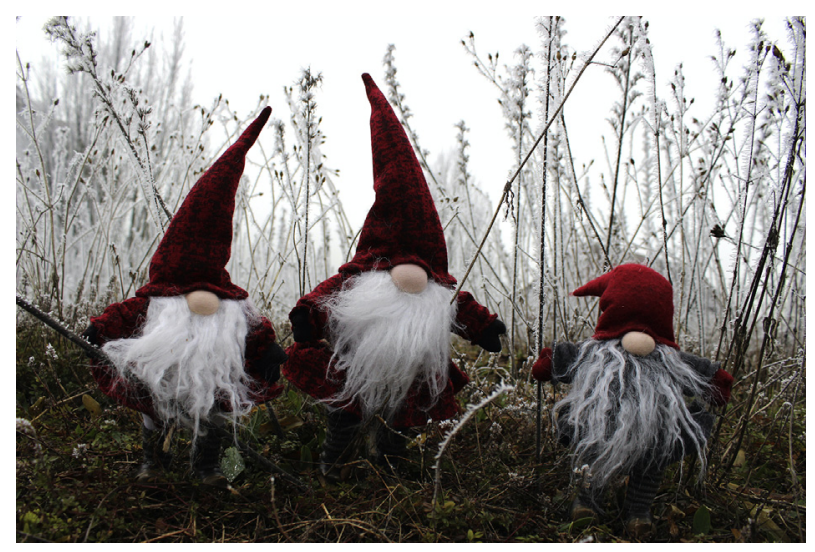

FIGURE 1. A child can sometimes seem like a small adult or an adult can act like a small child. However, specific parameters should be used in pediatric populations to achieve the best medical and surgical management.

indications and rates of reinterventions using these new parameters must also be completed. Although these results come from an elegant study with sophisticated statistical analysis, the duty of the authors will be now to spread the word. We are all ears!

\section{References}

1. Moore P. Children are not small adults. Lancet. 1998;352:630. https: //doi.org/10.1016/S0140-6736(05)79591-X

2. Ahmed Bhimani S, Rahmy A, Kim S, Jin JB, Zahka K, Komarlu R, et al. Optimizing evaluation in pediatric and young adult Marfan syndrome: novel longitudinal metrics to track growth of aortic structures. J Thorac Cardiovasc Surg. 2022; 164:724-40.e6. 